\title{
"A tough sell": findings from a qualitative analysis on the provision of healthy foods in recreation and sports settings
}

\author{
Jessie-Lee D. Mclsaac, PhD (1); Sherry L. Jarvis, MA (1); Rebecca Spencer, MA (2); Sara F.L. Kirk, PhD (1,2)
}

Tweet this article

\begin{abstract}
Recreation and sport settings (RSS) typically promote health in the form of physical activity, but the healthfulness of their food environment is often neglected. We explored stakeholder perspectives on barriers to healthy food provision in RSS through telephone interviews with ten representatives from RSS across Nova Scotia. Three key barriers were identified: 1) cultural norms associated with food in RSS and the broader environment, 2) the persisting notion of personal choice and responsibility, and 3) financial implications of healthy food provision. These barriers challenge healthy food provision in RSS and require multi-faceted strategies to overcome social norms that undermine health behaviours.
\end{abstract}

Keywords: food culture, health promotion, healthy eating, nutrition, recreation, sports, policy

\section{Introduction}

Around three-quarters of children in Canada participate in organized sports like soccer and swimming. ${ }^{1}$ Recreation and sport settings (RSS) - a term that includes multi-purpose fitness centers, arenas, rinks, and sports fields-provide opportunities for such structured physical activity, as well as modelling other health promotion foci like injury prevention or smoke-free spaces. ${ }^{2}$ Yet, paradoxically, the food environments within RSS often contradict overall health promotion messages through the provision of energy-dense, nutrient-poor foods that are quick to prepare and inexpensive to provide, yet profitable. ${ }^{3-8}$ This apparent contradiction presents an opportunity for RSS to make improvements to their food environments to support health behaviours of the families that use them. ${ }^{?}$

Research from other provinces in Canada has identified barriers to healthy food provision within RSS, including financial concerns, the current food culture, and difficulty getting stakeholder buy-in ${ }^{3-6}$ for changes in food provision. With the province of Nova Scotia having among the highest rates of chronic disease in Canada ${ }^{10}$, and prior to the release of voluntary guidelines to support healthy eating in RSS (HERSS guidelines) within the province ${ }^{11}$, we sought to qualitatively describe the barriers present within provincial RSS that may impact uptake of the HERSS guidelines prior to their release in October 2015.

\section{Methods}

We used Qualitative Description (QD) to describe the food provision experiences of ten key stakeholders from provincial RSS, as described in Table 1. Less interpretative than other methodologies, QD stays close to the data, permitting a summary of events "in the everyday terms of those events." 12 Participants were recruited from RSS via email using purposive sampling that focussed on those with the most insight into the factors that influences food provision within their RSS, such as facility or recreation managers. ${ }^{13}$ The Dalhousie University Research Ethics Board approved this study.

\section{Highlights}

- Cultural norms and values related to food provision in recreation and sport settings risk limiting the impact of interventions designed to support healthy food provision.

- Perceived opposition from patrons and accompanying concerns regarding loss of revenue among stakeholders are persisting obstacles, reinforced by a societal narrative that promotes personal choice as a primary driver for food provision.

- A multifaceted approach is needed to address the complexity of changing social norms and promoting healthy choices in recreation and sport settings.

Data collection involved individual semistructured telephone interviews, lasting 45 to 60 minutes, on stakeholder experiences with food provision in RSS and perceptions about implementing healthy eating policies and practices. Data were collected between August and October 2014 and ended when no new data were emerging from interviews. Qualitative analysis software (QSR NVivo Version 10) was used for management and analysis. Open coding was conducted to inductively identify and define emerging codes, and an iterative approach was used. ${ }^{14,15}$ Two authors (Jarvis, Spencer) developed the codes, portions of interviews were coded by each author to allow comparison, and all authors contributed to theme development to enhance credibility, authenticity, and confirmability. ${ }^{12,16,17}$ 
TABLE 1

\section{Participant characteristics}

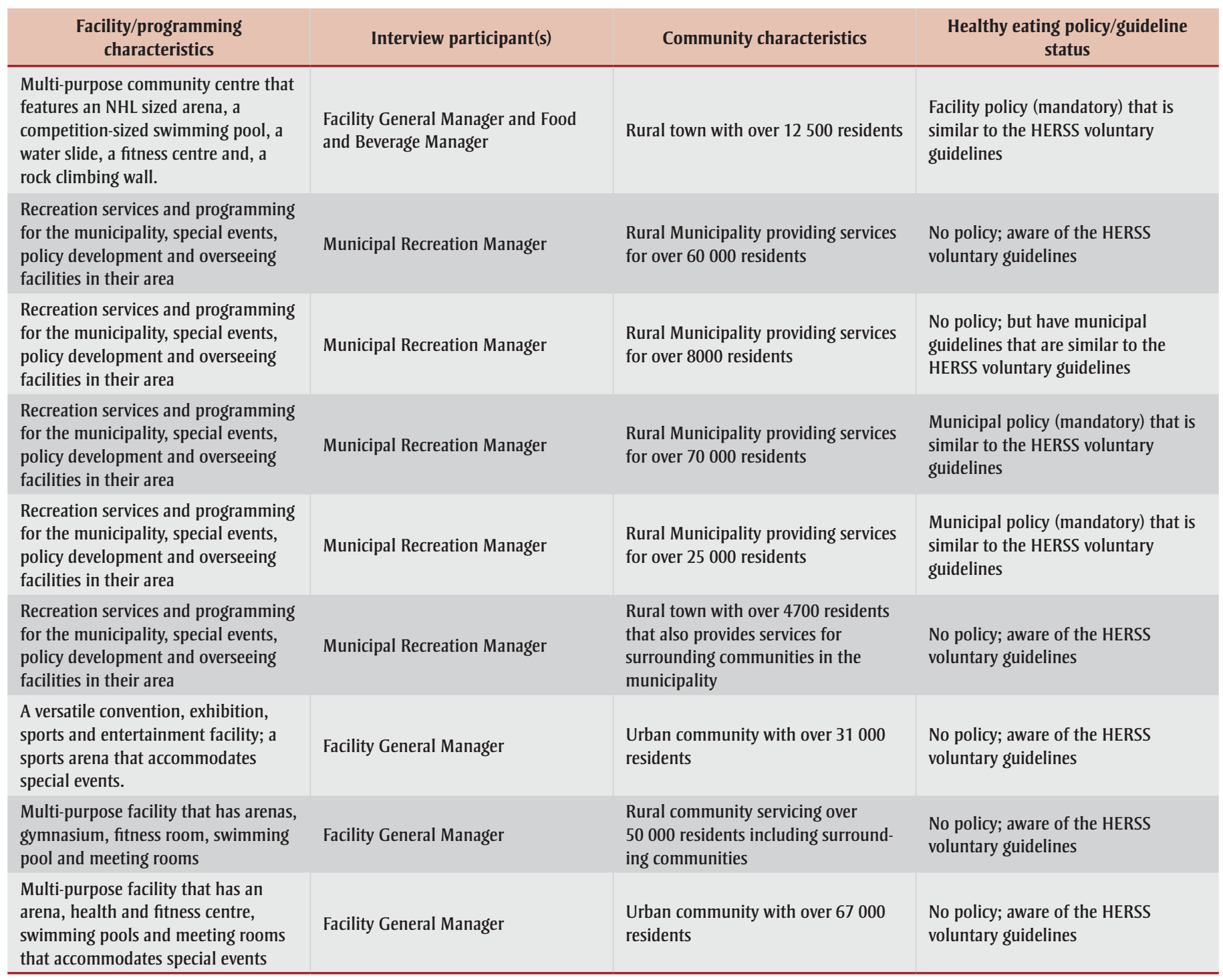

Abbreviations: HERSS, healthy eating in recreation and sport settings; NHL, National Hockey League.

\section{Results}

Three key themes emerged related to the implementation of healthy eating within RSS (quotes presented in Table 2): 1) cultural norms associated with food in RSS and the broader environment; 2) the persisting notion of personal choice and responsibility; and 3) financial implications of healthy food provision. A summary of each theme is provided below.

\section{Cultural norms associated with food in RSS and the broader environment}

This was the most predominant theme, represented as the social norms and patron expectations of the types of foods that should be available within RSS.
Unhealthy food traditions were viewed as being associated with certain sports, e.g. hockey and baseball, resulting in resistance to change toward healthier options, for fear that patrons would be unwilling to purchase them. The belief that patrons expected less healthy foods to be available at special events was typically framed within the context of being an occasional "treat." There were some types of sports, however, e.g. figure skating, dancing and soccer, that were perceived to be more open to healthier options being provided.

\section{The persisting notion of personal choice and responsibility}

This theme is illustrated through the tension participants described between supporting personal choice through food provision and the responsibility of RSS to be leaders in changing food environments. Most participants felt that staff within RSS should not be telling people what they can and cannot eat. Willingness to offer more healthful items was framed as being acceptable only alongside less healthy options, to allow patrons to make their own choice. The personal beliefs of participants about choice emerged in relation to their perception of the role RSS should play in creating healthy food environments. Participants expressed concerns that changes to the food environment to support health may be beyond the mandate of RSS. Rather than individual RSS being responsible for initiating changes to 
TABLE 2

Themes and illustrative quotes

Theme

Cultural norms associated with food in RSS and the broader environment

The persisting notion of personal choice and responsibility

Financial implications of healthy food provision
Quotes

“Baseball is probably the biggest barrier for nutritional change, uh, due to uh, I don't know, nostalgia, or food related items that are just attached to baseball culture such as hot dogs and chips and pop and pizza and peanuts and sunflower seeds."

“People weren't buying into it...the majority of people that come through here are hockey, hockey minded people...it's just, that's, that's the culture that's built around uh, around the sports...I mean, the NHL players, you see them on TV and they're promoting [sports drinks], and uh, and [Brand] Chips."

“...like a big issue with the rinks is their fries. They love their fries... so, for me to tell them that they can't have fries anymore, well we might as well just close the canteen and shut the rink down, that's the end of it."

"Like everyone's like, well celebrations are important, and we should have celebrations and there has to be, like, junk food at that function. Like, there just seems to be a real, a real unwillingness for a lot of people to just let go of that."

“...yeah, hockey, hockey culture is certainly different than uh, say the figure skating.... Well, I mean there's a number of reasons uh, but it's just, it's a different sport, it's just like running is to uh, baseball. Uh, it's a different mindset...."

"I think people should be able to make the choice.... that's what I believe. I don't think the arena, okay, is the place, I don't think we're the ones responsible for making that decision for people. I think people have to be informed, you have to educate them on, they'll make that decision for me."

"...the provincial body of each sport has to instill that into their programmers, coaching staff and so on and so forth and then that has to be, because uh, I can only do so much as a facilitator, as a manager of a facility..."

“... I don't think, most people who come to the hockey games are sitting down eating a box of poutine every night that they're home. So they come here and that's their treat, okay? ...So, I think, if I went the other way, I would see my revenue drop."

"And the marketplace will dictate everything...like, once the customer decides that, I'm not going to buy your French fries and hot dogs, then you won't be selling French fries and hot dogs."

“...selling something that's going to create a healthier community versus something that's going to cost, that could lose money, is a tough sell...people look at dollar value versus health value unfortunately."

“...last year I gave it a real good kick at the can and the last year I hired an individual who's a very good chef ... and boy, we gave it a real good shot but it uh, it just, it failed miserably."

Abbreviations: NHL, National Hockey League; RSS, recreation and sports settings.

support healthy eating, some participants felt that provincial sport or recreation organizations that provide overall direction for sport groups and facilities should be involved in leading change.

\section{The financial implications of healthy food provision}

This third theme represents the fear expressed by participants, particularly facility managers, that revenue would be lost if they sold healthier foods and/or stopped selling less healthy foods. Framed within the cultural context that less healthy foods were a "treat", despite their ubiquity, their provision was attributed to patrons wanting the less healthy options. Healthy foods were viewed as more costly, requiring more preparation time and different storage needs, and that waste would occur if the food did not sell. This was particularly salient for facilities that were not open every day, e.g. some arenas.

\section{Discussion}

Our findings reinforce the challenges facing health promoters in creating environments that support healthy behaviours. Allowing personal choice to dictate food provision, while preferred by participants in this analysis, has been found to limit the impact of changes designed to improve healthy food provision, which is particularly concerning in settings frequented by children and youth. ${ }^{6}$ When healthy and unhealthy choices are available within RSS, children and youth continue to purchase primarily unhealthy options. ${ }^{6,18}$ Further, our current unhealthy food culture makes the notion of personal choice a complex one because many social norms, learned behaviours, and expectations about food are strongly influenced by marketing campaigns of the food industry. ${ }^{19,20}$ Adults are equally susceptible to selecting unhealthy foods, or wanting unhealthy foods such as hot dogs and fries to be readily available in RSS facilities. $^{21}$ This further highlights how the availability of unhealthy foods in settings like RSS is both normalized and entrenched. ${ }^{22}$

Consistent with other Canadian studies, participants perceived healthy foods to be more expensive to purchase, prepare and store, and overall less marketable than unhealthy foods. ${ }^{2,4,5,23}$ Emerging evidence from a recent intervention study challenges this perception ${ }^{24}$. In this intervention, resources were provided to an intervention group to help them change the food and beverage environment within sporting clubs, while the control group received no support. No significant differences in annual revenues were observed following the intervention between the control and intervention sporting clubs, ${ }^{24}$ indicating that the intervention group did not lose revenue as a result of selling 
healthier options. This has also been found in research from Alberta. $^{23}$ Unhealthy food environments in RSS may be paradoxically an unintended consequence of improving accessibility to physical activity participation ${ }^{25}$ with user fees for RSS services often being subsidized by food service revenues that are, in turn, heavily dictated by the social norms of RSS settings. ${ }^{23}$ Ideally, physical activity and healthy eating should not be competing priorities in RSS to support overall health promotion goals but there remain obstacles to overcome the deeply entrenched values associated with food.

A strength of our study is the participation of stakeholders across different regions of Nova Scotia and diverse types of facilities with varying experiences of healthy eating initiatives. During the final stages of interviews and analysis there were no new emerging themes that developed, which suggested that we had sufficient representation of perspectives from stakeholders in the province. However, one limitation was the use of telephone interviews, as we may have missed important non-verbal cues that could have contributed to the analysis.

\section{Conclusion}

Our findings reinforce the need to address social norms that undermine health behaviours. This requires champions who can advocate for healthy eating and take action to overcome resistance to healthy eating guidelines. ${ }^{5}$ Health promotion strategies that may support the implementation of healthy eating guidelines should also provide social supports, such as training and resources, and strategies that increase the availability and promotion of healthy foods, while limiting the marketing and advertising of unhealthy foods. ${ }^{2,26}$ Emerging evidence reveals that interventions using a combination of these strategies have a greater impact on healthy eating choices and behaviours. ${ }^{17,24}$ It is clear that a multifaceted approach is critical to address the complexity of changing social norms and promoting healthy choices in RSS. ${ }^{21,27}$ Changes to food environments within RSS as a result of voluntary guidelines implementation will likely only be modest, ${ }^{5}$ without greater attention to perceptions surrounding the cost of healthy foods and the social norms that continue to shape unhealthy food provision in these settings.

\section{Acknowledgements}

The authors would like to thank all participants from recreation and sport settings in Nova Scotia for their support of this research.

This research was supported by the Nova Scotia Department of Health and Wellness, the Heart and Stroke Foundation of Canada and the Canadian Institutes of Health Research (CIHR) FRN 134228. McIsaac, J-L acknowledges funding from the Canadian Cancer Society (grant \# 703878). Jarvis, S received salary support from the Heart and Stroke Foundation of Canada. Spencer, R acknowledges funding from a CIHR Doctoral Research Award. Kirk, SFL held a CIHR-funded Canada Research Chair at the time this study was conducted.

\section{Conflicts of interest}

The authors had no conflicts of interest to report.

\section{Authors' contributions and statement}

SFLK and JDM conceived and designed the study. SJ collected the data and SJ and RS conducted the data analysis. All authors drafted and wrote parts of the manuscript, were involved in the interpretation of data and critically revising the manuscript. All authors read and approve the final manuscript.

The content and views expressed in this article are those of the authors and do not necessarily reflect those of the Government of Canada.

\section{References}

1. Tremblay MS, Barnes JD, Gonzalez SA, et al. Global Matrix 2.0: Report card grades on the physical activity of children and youth comparing 38 countries. Journal of Physical Activity and Health. 2016;13 (11):S343-66. doi: 10.1123/jpah.2016-0594.

2. Dobbinson SJ, Hayman JA, Livingston PM. Prevalence of health promotion policies in sports clubs in Victoria, Australia. Health Promot Int. 2006 Jun;21(2):121-9. doi: 10 .1093 /heapro/dak001.
3. Naylor P-J, Bridgewater L, Purcell M, Ostry A, Wekken SV. Publically Funded Recreation Facilities: Obesogenic Environments for Children and Families? Int J Environ Res Public Health. 2010 May;7(5):2208-21. doi: 10.3390/ijerph7052208.

4. Naylor P-J, Vander Wekken S, Trill D, Kirbyson A. Facilitating Healthier Food Environments in Public Recreation Facilities: Results of a Pilot Project in British Columbia, Canada. J Park Recreat Adm. 2010;28(4):37-58.

5. Olstad DL, Lieffers JRL, Raine KD, McCargar LJ. Implementing the Alberta nutrition guidelines for children and youth in a recreational facility. Can J Diet Pract Res. 2011;72(4): 177. doi: 10.3148/72.4.2011.e212.

6. Olstad DL, Raine KD, McCargar LJ. Adopting and implementing nutrition guidelines in recreational facilities: Public and private sector roles. A multiple case study. BMC Public Health. 2012;12:376.

7. Priest N, Armstrong R, Doyle J, Waters E. Policy interventions implemented through sporting organisations for promoting healthy behaviour change. In: The Cochrane Collaboration, editor. Cochrane Database of Systematic Reviews [Internet]. Chichester, UK: John Wiley \& Sons, Ltd; 2008 [cited 2016 May 19]. doi: 10.1002/14651858 .CD004809.pub3.

8. Thomas HM, Irwin JD. Food choices in recreation facilities: operators' and patrons' perspectives. Can J Diet Pract Res. 2010;71(4):180-5. doi: 10 .3148/71.4.2010.180.

9. World Cancer Research Fund International. NOURISHING framework [Internet]. Available from: http:// www.wcrf.org/int/policy/nourishing -framework

10. Province of Nova Scotia. Thrive! A plan for a healthier Nova Scotia [Internet]. Available from: https:// thrive.novascotia.ca/

11. Province of Nova Scotia. Healthy eating in recreation and sport settings guidelines [Internet]. Retrieved from https://thrive.novascotia.ca/node /8311 
12. Neergaard MA, Olesen F, Andersen RS, Sondergaard J. Qualitative description - the poor cousin of health research? BMC Med Res Methodol. 2009 Jul 16;9(1):52. doi: 10.1186/1471 $-2288-9-52$.

13. Sandelowski M. Whatever happened to qualitative description? Res Nurs Health. 2000 Aug;23(4):334-40. doi: $10.1002 / 1098-240 X(200008) 23: 4$ \%3C334::AID-NUR9\%3E3.0.CO;2-G.

14. Patton MQ. Qualitative Research and Evaluation Methods. 3rd ed. Thousand Oaks, CA: Sage Publications; 2002.

15. Miles MB, Huberman AM. Qualitative Data Analysis: An expanded sourcebook. 2nd ed. Thousand Oaks, CA: Sage Publications; 1994.

16. Milne J, Oberle K. Enhancing rigor in qualitative description: a case study. J Wound Ostomy Cont Nurs Off Publ Wound Ostomy Cont Nurses Soc WOCN. 2005 Dec;32(6):413-20. doi: 10.1097/00152192-200511000-00014.

17. Morrow SL. Quality and trustworthiness in qualitative research in counseling psychology. J Couns Psychol. 2005;52(2):250-60. doi: 10.1037/0022-0167.52.2.250.

18. Van Hulst A, Barnett TA, Déry V, Côté $\mathrm{G}$, Colin C. Health-promoting vending machines: evaluation of a pediatric hospital intervention. Can J Diet Pract Res. 2013;74(1):28-34. doi: 10.3148 /74.1.2013.28,

19. Elliott C. "It's junk food and chicken nuggets": Children's perspectives on "kids" food' and the question of food classification. J Consum Behav. 2011; 10(3):133-40.

20. Ludwig DS, Nestle M. Can the food industry play a constructive role in the obesity epidemic? JAMA. doi: 10.1001/jama.300.15.1808.

21. Olstad DL, Goonewardene LA, McCargar LJ, Raine KD. Choosing healthier foods in recreational sports settings: a mixed methods investigation of the impact of nudging and an economic incentive. Int $\mathrm{J}$ Behav Nutr Phys Act. 2014;11:6. doi: 10.1111/j .1468-0009.2009.00550.x.

22. Sallis JF, Glanz K. Physical activity and food environments: solutions to the obesity epidemic. Milbank Q. 2009;87(1):123-54. doi: 10.1111/j.1468 $-0009.2009 .00550 . x$.
23. Olstad DL, Downs SM, Raine KD, Berry TR, McCargar LJ. Improving children's nutrition environments: a survey of adoption and implementation of nutrition guidelines in recreational facilities. BMC Public Health. 2011;11:423. doi: 10.1186/1471-2458 $-11-423$.

24. Wolfenden L, Kingsland M, Rowland BC, Dodds P, Gillham K, Yoong SL, et al. Improving availability, promotion and purchase of fruit and vegetable and non sugar-sweetened drink products at community sporting clubs: a randomised trial. Int $\mathrm{J}$ Behav Nutr Phys Act. 2015;12:35. doi: 10.1186 /s12966-015-0193-5.

25. Olstad DL, Raine KD. Profit versus public health: the need to improve the food environment in recreational facilities. Can J Public Health. 2013; 104(2):e167-169.

26. Social Research and Demonstration Corporation. Evaluation of the Healthy Food and Beverage Sales in Recreation Facilities and Local Government Buildings Initiative [Internet]. Victoria, BC: University of Victoria; 2010 [cited 2016 May 19]. Available from: http://www.srdc.org /uploads/BCHLA_HealthyFood.pdf

27. Chan RSM, Woo J. Prevention of overweight and obesity: how effective is the current public health approach. Int J Environ Res Public Health. 2010 Mar;7(3):765-83. 